\title{
Motor Unit Properties and Recruitment in the Larval Zebrafish
}

\author{
Timothy Wiggin \\ Department of Neuroscience, University of Minnesota-Twin Cities, Minneapolis, Minnesota 55455 \\ Review of Menelaou and McLean
}

One important task for all animals is translating the intention to move into action by contraction or relaxation of the appropriate muscle groups. The smallest unit of voluntary muscle activation is a single motor neuron and the muscle fibers that it innervates, collectively called a "motor unit" (Liddell and Sherrington, 1925). Motor units translate neuronal activity into a mechanical force appropriate to the desired action. The force produced by a motor unit is primarily controlled by the size and spike frequency of the motor neuron. According to the size principle, the size of the motor neuron is correlated with the force produced by its activity (Mendell, 2005). The smallest motor neurons innervate the weakest muscle fibers and are recruited during the least forceful contractions. With increasing total contractile force, progressively larger motor neurons, which innervate stronger muscle fibers, are recruited (Clamann, 1993). Within the recruitment range of each motor unit, motor neuron spike frequency is correlated with the tension exerted by the innervated muscle fiber, allowing each motor unit to produce a graded force output (Clamann, 1993).

Received Sept. 25, 2012; revised 0ct. 19, 2012; accepted 0ct. 24, 2012.

T.W. is supported by National Institutes of Health Grant R01 NS065054.

Many thanks to Mark A. Masino, Jack H. Peck, and Jacob E. Montgomery for helpful comments on the manuscript.

Correspondence should be addressed to Timothy Wiggin at the above address. E-mail: wiggi063@umn.edu.

DOI:10.1523/JNEUROSCI.4575-12.2013

Copyright $\odot 2013$ the authors $\quad 0270-6474 / 13 / 330853-02 \$ 15.00 / 0$
In both larval and adult zebrafish, the size principle is paired with a dorsoventral gradient of recruitment. Only ventral motor neurons are recruited during lowfrequency swimming, whereas both dorsal and ventral motor neurons are recruited during high-frequency swimming (McLean et al., 2007; Gabriel et al., 2011). Gabriel et al. (2011) found that intrinsic membrane properties of motor neurons and the size of their synaptic currents varied along the dorsoventral axis in adult zebrafish. But because motor unit properties could not be linked to motor neuron membrane properties in their decerebrate adult zebrafish preparation, only indirect inferences about the behavioral consequences of motor neuron properties could be made. In addition, this preparation is limited in that it swims only after chemical or electrical stimulation and it does not produce the full range of behaviors of the intact animal.

In a recent paper published in The Journal of Neuroscience, Menelaou and McLean (2012) used intact larval zebrafish to bridge the gap between motor neuron and motor unit properties by quantifying the dorsoventral position, innervation pattern, and intrinsic membrane properties of motor neurons, and correlating these properties with motor neuron activity during fictive swimming. The large degree of agreement between Menelaou and McLean (2012) and Gabriel et al. (2011) confirms that there are significant similarities between the motor pools of larval and adult zebrafish, and al- lows inference between the respective developmental stages using the strengths of each.

To investigate the relationship between motor neuron soma position and innervation pattern, Menelaou and McLean (2012) filled motor neurons with fluorescent dye and traced their projections into the axial muscle. Consistent with the size principle, the largest motor neurons primarily innervated fast muscle layers and were restricted to the dorsal region of the motor neuron pool, while smaller motor neurons were located more ventrally and primarily innervated slow muscle fibers (Menelaou and McLean (2012), their Fig. 4). Therefore, both soma size and position of motor neurons were correlated with the putative strength of the muscle fibers that they innervated.

To investigate the mechanism of orderly recruitment, Menelaou and McLean (2012) measured the membrane properties and recruitment of motor neurons over a wide range of fictive swimming frequencies using whole-cell patch-clamp recordings. Although the range of electrophysiological properties of motor neurons might best be described as a continuum, the authors categorize neurons into three distinct phenotypes (tonic, chattering, and bursting) to quantify their traits and determine their overlap with morphologically defined motor neuron types.

The tonic class was characterized by a high spike threshold and high-frequency tonic spiking with limited spike frequency 
adaptation in response to suprathreshold current injection. All so-called primary motor neurons (large, dorsal, earlydeveloping neurons) that were recorded belonged to the tonic class and all tonic neurons were primary motor neurons. Chattering motor neurons were characterized by a lower spike threshold than the tonic class, and they showed significant spike frequency adaptation (with occasional recovery) during prolonged suprathreshold current injection. The bursting class of motor neurons had the lowest spike threshold, and produced slow membrane potential changes that led to bursts of action potentials separated by subthreshold intervals during sustained current injection. There were no exclusive correspondences between the chattering or bursting electrophysiological classes and any morphological classes of motor neurons. Despite this lack of categorical separation, bursting motor neurons tended to be more ventral than chattering motor neurons (Menelaou and McLean (2012), their Fig. 6).

In addition to injecting current into motor neurons during non-swim periods, Menelaou and McLean (2012) observed the activity of motor neurons during swimming. Consistent with the size principle and dorsoventral recruitment order, burst neurons were recruited at the lowest swim frequencies, followed by chattering, and finally tonic motor neurons. During swim cycles in which the amplitude of the membrane potential oscillation failed to reach spike threshold, the magnitude of the oscillation increased with increasing swimming frequency in tonic and chattering, but not in burst motor neurons. This difference in the dynamic range of the subthreshold oscillation amplitude observed may reflect the mechanism that drives the dorsoventral gradient of motor neuron recruitment, but there are several potential explanations for this phenomenon.

One possibility is that chattering and tonic neurons receive a greater number of excitatory synapses and therefore show a greater range of synaptic input than the bursting neurons. This would be consistent with adult zebrafish motor neurons in which the amplitude of synaptic input is correlated with recruitment order (Gabriel et al., 2011). A second possibility is that bursting motor neurons have voltage-sensitive channels that amplify synaptic current, masking changes in the magnitude of synaptic input across swimming frequencies. Amplification of synaptic input by motor neurons has been observed in mammals (Jones and Lee, 2006), and the slow membrane changes in bursting motor neurons could be due to a voltage-sensitive depolarizing intrinsic membrane current. A third possibility is that the bursting motor neuron class receives both increasing excitatory and increasing inhibitory synaptic input with higher swimming frequencies. This would be similar to the mechanisms that regulate the premotor interneurons that drive slow swimming (McLean et al., 2008) and would explain the authors' finding that a subset of the burst motor neuron population becomes less reliably recruited at higher swimming frequencies (Menelaou and McLean (2012), their Fig. 7).

Similar to findings in adult zebrafish (Gabriel et al., 2011), Menelaou and McLean (2012) report a class of larval motor neurons that exhibit a bursting phenotype during constant current injection. The interpretation that the authors offer is that bursting is evidence for intrinsic rhythmicity in the bursting motor neurons, and that the rhythmicity contributes to shaping the motor output of the cell during swimming. This view is supported by several lines of reasoning. First, the long latency to the first spike and slow rise in membrane potential in the bursting cells following current injection (Menelaou and McLean (2012), their Fig. 5) is suggestive of a slow, voltage-activated, depolarizing intrinsic membrane current. Second, bursts self-terminate without a change in the current injection, which could be explained by the activation of a delayed, hyperpolarizing membrane current. Third, when the larval zebrafish is not swimming, there is very little spontaneous synaptic input to the motor neuron pool, which suggests that their spiking behavior during current injection does not depend on the spinal locomotor network.

However, each of these lines of evidence is confounded by the ability of the motor neurons to make synaptic contact with other neurons during current injection. The slow depolarization observed in bursting cells could result from synaptic input from an electrically coupled neuron that was brought above firing threshold by the current injection and had an excitatory chemical synapse onto the patched neuron. Self-termination of bursts could be explained by feedback-inhibitory input from a Renshaw-like neuron (Curtis and Ryall, 1964), and the oscillation frequency could be explained by latency due to a muscarinic synapse (McCormick and Prince, 1985). Finally, while the spinal lo- comotor network is inactive when the larval zebrafish is not swimming, the authors demonstrate that motor neurons have intraspinal collaterals (Menelaou and McLean, 2012, their Fig. 3) that may make synaptic contact with other neurons and activate small neuronal networks.

The authors' findings have important implications for the study of locomotion. The authors provided a detailed map of the anatomical and electrophysiological properties of the motor neuron pool in the larval zebrafish, which will guide future studies in the mechanisms of larval zebrafish steering and prey capture. The authors also found that, even at larval stages, zebrafish motor neurons exhibit a broad continuum of electrophysiological properties. While the basis for these properties remains unclear, their early appearance and persistence into adulthood suggest that they play an important role in shaping behavior.

\section{References}

Clamann HP (1993) Motor unit recruitment and the gradation of muscle force. Phys Ther 73:830-843. Medline

Curtis DR, Ryall RW (1964) Nicotinic and muscarinic receptors of Renshaw cells. Nature 203:652-653. CrossRef Medline

Gabriel JP, Ausborn J, Ampatzis K, Mahmood R, Eklöf-Ljunggren E, El Manira A (2011) Principles governing recruitment of motoneurons during swimming in zebrafish. Nat Neurosci 14:93-99. CrossRef Medline

Jones SM, Lee RH (2006) Fast amplification of dynamic synaptic inputs in spinal motoneurons in vivo. J Neurophysiol 96:2200-2206. CrossRef Medline

Liddell EG, Sherrington CS (1925) Recruitment and some other features of reflex inhibition. Proc R Soc Lond B Biol Sci 97:488-518. CrossRef

McCormick DA, Prince DA (1985) Two types of muscarinic response to acetylcholine in mammalian cortical neurons. Proc Natl Acad Sci U S A 82:6344-6348. CrossRef Medline

McLean DL, Fan J, Higashijima S, Hale ME, Fetcho JR (2007) A topographic map of recruitment in spinal cord. Nature 446:71-75. CrossRef Medline

McLean DL, Masino MA, Koh IY, Lindquist WB, Fetcho JR (2008) Continuous shifts in the active set of spinal interneurons during changes in locomotor speed. Nat Neurosci 11: 1419-1429. CrossRef Medline

Mendell LM (2005) The size principle: a rule describing the recruitment of motoneurons. J Neurophysiol 93:3024-3026. CrossRef Medline

Menelaou E, McLean DL (2012) A gradient in endogenous rhythmicity and oscillatory drive matches recruitment order in an axial motor pool. J Neurosci 32:10925-10939. CrossRef Medline 